\title{
HPLC/APCI-FTICR-MS as a Tool for Identification of Partial Polar Mutagenic Compounds in Effect-Directed Analysis
}

\author{
Mahmoud Bataineh, ${ }^{\text {a,b }}$ Urte Lübcke-von Varel, ${ }^{a}$ Heiko Hayen, ${ }^{\mathrm{c}}$ and \\ Werner Brack ${ }^{\mathrm{a}}$ \\ ${ }^{a}$ Department of Effect-Directed Analysis, UFZ-Helmholtz Centre for Environmental Research, Leipzig, \\ Germany \\ ${ }^{\mathrm{b}}$ Department of Chemical Engineering, Abu Dhabi Men's College, Abu Dhabi, United Arab Emirates \\ ${ }^{\mathrm{c}}$ Leibniz-Institut für Analytische Wissenschaften -ISAS-e.V., Dortmund, Germany
}

Identification of unknown compounds remains one of the biggest challenges for the assignment of adverse effects of sediment contamination and other complex environmental mixtures to responsible toxicants by effect-directed analysis (EDA). The identification depends on information gained from biotesting, chromatographic separation, and mass spectrometric detection. Thus, a methodology is provided for non-target identification of partial polar mutagenic polyaromatic compounds in sediment extracts by using polymeric reversed-phase HPLC column, high-resolution mass spectrometry and PubChem database. After visualization and processing the chromatogram constituents by using deconvolution software, the unambiguous elemental compositions generated were used as input in PubChem database to find a possible identity for the suspected species. The retrieved structures from the database search were refined by characterized chromatographic and mass spectrometric classifiers based on 55 model compounds comprising eight different classes representing mutagenic substructures. The applicability of the method was demonstrated by positive and tentative identification of constituents of mutagenic sediment fractions similar to selected model compounds. (J Am Soc Mass Spectrom 2010, 21, 1016-1027) (C) 2010 American Society for Mass Spectrometry

$\mathrm{A}$ quatic environments are contaminated with a multitude of organic compounds. Although most of them occur in trace concentrations, they may affect aquatic ecosystems and biodiversity due to their toxic potency [1]. Polycyclic aromatic compounds (PACs), including polycyclic aromatic hydrocarbons (PAHs) and a multitude of nitro, amino, keto, hydroxyl, quinone, and heterocyclic derivatives thereof are frequent contaminants in aquatic sediments and suspicious as the cause of adverse effects to biota, including mutagenicity, carcinogenicity, endocrine disruption, and developmental effects [2,3]. Effect-directed analysis (EDA) aims to identify the compounds causing these effects after reducing sample complexity by combining biotests with fractionations [4]. This methodology was found to be a powerful tool to support the establishment of cause-effect relationships between contamination and effects to aquatic organisms $[5,6]$. However, structure elucidation of unknown compounds in isolated toxic fractions remains one of the biggest challenges to the successful completion of EDA, since these fractions often remain rather complex even after extensive fractionation. The amounts and purity of the com-

Address reprint requests to Dr. M. Bataineh, Department of Chemical Engineering, Abu Dhabi Men's College, Abu Dhabi, United Arab Emirates. E-mail: mbataineh@hct.ac.ae or mhmoudb@yahoo.com pounds present $[7,8]$ are often not sufficient for analysis using nuclear magnetic resonance spectroscopy or infrared spectroscopy, and thus the toxic fractions are usually analyzed by chromatography hyphenated with mass spectrometric detection. In most cases, gas chromatography coupled to mass spectrometry (GC-MS) is applied [9-22]. The major disadvantage of GC-MS is the requirement for compound volatility and thermal stability, which limits the range of compounds that can be analyzed successfully. HPLC-based methods have increasingly gained importance for structure elucidation. However, the lack of easy-to-use spectral libraries for compound identification [8] demand for the development of alternative approaches for structure elucidation exploiting as much information as possible gained from chromatographic retention and MS. Recently, the development of atmospheric pressure ionization (API) and high-resolution (HR) MS has opened new opportunities in chemical identification of unknowns, with techniques such as Fourier transform ion-cyclotron resonance- (FTICR) MS and quadrupole time of flight- (Q-TOF) MS providing mass accuracies of better than 5 ppm [23-29]. Based on the accurate mass and the isotope pattern, high-resolution MS software generates accurate empirical formulas for low molecular weight compounds. These formulas are ready for search in comprehensive compound databases (i.e., PubChem, Merck index). Moreover, 
the use of classifiers helps to reduce the number of hits and facilitates tentative identification [30-33].

The aim of the present study was to develop a methodology for identification of partial polar sedimentassociated PACs in the frame of EDA. Method development was based on 55 model compounds applying HPLC on polymeric RP-C18 stationary phase and atmospheric pressure chemical ionization (APCI)-FTICRMS/MS. The method was evaluated by the analysis of partial polar mutagenic sediment extract samples from the river Elbe basin (Germany). The chromatogram constituents were visualized and processed by adapting an open source metabolomics deconvolution software (MZmine) [34-36]. The resulting empirical formulas were searched in PubChem database and the retrieved hits were refined based on the characterized diagnostic classifiers from APCI-MS/MS and chromatographic behavior for tentative identifications.

\section{Experimental}

\section{Chemicals and Materials}

Methanol for the chromatographic separation was purchased from Carl Roth (Karlsruhe, Germany), and highpurity water was taken from a Seral-Pur Delta UV apparatus (USF Seral, Ransbach, Germany). Chemical standards were purchased from several commercial suppliers as listed in Table S-1 in the Supplementary Information, which can be found in the electronic version of this article. Three mutagenic fractions labeled B14-6-10 (F1), B14-10-8 (F2), and B15-9-9 (F3) from a sediment extract were provided for method evaluation by an EDA study [37, 38].

\section{Analysis of PACs by Liquid Chromatography-Mass Spectrometry (LC-MS)}

The fractionated extracts were dissolved in methanol before analysis. Chromatographic separations were performed using a Surveyor MS pump and Surveyor autosampler (Thermo Fisher Scientific, San Jose, CA, USA) and polymeric reversed-phase column (Supelcosil LC-PAH, $250 \times 2.1 \mathrm{~mm}, 5 \mu \mathrm{m}, 120 \AA$; Supelco, Deisenhofen, Germany). Sample volumes of $5 \mu \mathrm{L}$ were injected. Compounds were separated at $40^{\circ} \mathrm{C}$ and a flow rate of $200 \mu \mathrm{L} / \mathrm{min}$ using a gradient of water containing methanol (5\%, vol/vol) (A) and pure methanol (B) as mobile phase according to the following elution program: $0-2$ min $40 \% \mathrm{~B}$, ramped to $100 \%$ B by $36 \mathrm{~min}$ (2-38), held for $8 \mathrm{~min}(38-46)$, ramped back to initial conditions in $2 \mathrm{~min}(46-48)$ and held for $10 \mathrm{~min}(48-58)$ to equilibrate the column before the next injection. Detection was performed by a hybrid linear ion trap (LIT)-FTICR-MS (Thermo Fisher Scientific, Bremen, Germany) equipped with an atmospheric pressure chemical ionization (APCI) source and controlled by Xcalibur software. Survey MS spectra in the mass range $m / z$ 100-400 were acquired in the FTICR with a resolu- tion $r=25,000$. The three most intense ions were sequentially isolated for accurate mass measurements by a FTICR "SIM scan" in a narrow mass window $( \pm 5$ $\mathrm{Da}, r=50,000)$. Subsequent tandem MS fragmentation was carried out in the LIT by collisionally induced dissociation (CID). Former target ions selected for $\mathrm{MS}^{2}$ were dynamically excluded for $45 \mathrm{~s}$. The general mass spectrometric conditions were: APCI source current, $5 \mu \mathrm{A}$ (pos.) and $10 \mu \mathrm{A}$ (neg.); $\mathrm{APCI}$ heater temperature, $450{ }^{\circ} \mathrm{C}$; sheath gas, 50 arbitrary units (au); auxiliary gas flow, 5 au and sweep gas flow, $5 \mathrm{au}$; ion transfer tube temperature, $300{ }^{\circ} \mathrm{C}$ and normalized collision energy $35 \%$ for $\mathrm{MS}^{2}$ with activation $q=0.25$ and activation time $30 \mathrm{~ms}$. Ion selection thresholds were 1000 counts. All data were processed using Qual Browser (Thermo Fisher Scientific, San Jose, CA, USA), and the chemical formula calculator was used for elemental composition determination.

\section{Chromatogram Visualization, Processing, Peak Selection, and Database Search}

To address the complexity of the chromatogram obtained, open source software MZmine (http:/ / mzmine. sourceforge.net) was used for visualization and processing of chromatograms constituents. To reduce the huge number of data to manageable numbers the noise level in MZmine was set to $5.0 \times 10^{3}$, the minimum peak height to $1 \times 10^{4}$, mass resolution to $25,000, \mathrm{~m} / \mathrm{z}$ tolerance to $0.002 \mathrm{u}$, and minimum peak duration to 0.2 min. For the custom database search, $\mathrm{m} / \mathrm{z}$ tolerance of $0.003 \mathrm{u}$ and a retention time tolerance of $30 \mathrm{~s}$ were used. MZmine software offered a quick processing tool by peak detection, calculating peak areas, and assignment of blank peaks by custom database search. After peak detection with MZmine and exclusion of blank $\mathrm{m} / \mathrm{z}$ values the remaining constituents containing $\mathrm{C}, \mathrm{H}, \mathrm{O}$, and $\mathrm{N}$ were selected for identification. The possible empirical formulas were determined by the QualBrowser, applying the following restrictions: $\mathrm{C}_{6-25}, \mathrm{H}_{0-30}, \mathrm{O}_{0-6}$, $\mathrm{N}_{0-6}$. The tentative identification was based on searching the generated empirical formulas after correction to the neutral mass in an open source PubChem database (http://pubchem.ncbi.nlm.nih.gov/search/search.cgi). PubChem database consists of more than 10 millions of records collected from different sources (EPA, Cambridge soft, etc.), and recently was used successfully for identification of biological compounds [30, 31] and herbal preparations [33] by narrowing the retrieved hits by certain classifiers (i.e., migration behavior, tandem MS).

\section{Results and Discussion}

\section{Analytical Method Development}

Method development was based on PACs with a wide range of functionalities using 55 compounds with less 
Table 1. Model substances investigated in this study with retention times, observed mass spectrometric signals, limits of detection, and tandem MS/MS data

\begin{tabular}{|c|c|c|c|c|c|c|}
\hline & $\mathrm{EF}$ & $\begin{array}{c}\mathrm{Rt} \\
{[\mathrm{min}]}\end{array}$ & Observed signal & $\mathrm{DL}(\mathrm{pg})$ & $\begin{array}{c}\text { MS/MS } \\
\text { (negative mode) }\end{array}$ & $\begin{array}{c}\text { MS/MS } \\
\text { (positive mode) }\end{array}$ \\
\hline \multicolumn{7}{|l|}{ Nitro-PAHs } \\
\hline 1-Nitronaphthalene & $\mathrm{C}_{10} \mathrm{H}_{7} \mathrm{NO}_{2}$ & 24.6 & {$\left[\mathrm{M}^{-}\right]^{-},[\mathrm{M}-\mathrm{NO}]^{-}$} & $25^{a} /$ n.d. ${ }^{b}$ & 143 & n.d. \\
\hline 2-Nitrofluorene & $\mathrm{C}_{13} \mathrm{H}_{9} \mathrm{NO}_{2}$ & 31.6 & {$[\mathrm{M}-\mathrm{H}]^{-},[\mathrm{M}-\mathrm{H}-\mathrm{NO}]^{-}$} & 50/n.d. & 180 & n.d. \\
\hline 9-Nitroanthracene & $\mathrm{C}_{14} \mathrm{H}_{9} \mathrm{NO}_{2}$ & 32.8 & {$[\mathrm{M}]-,[\mathrm{M}-\mathrm{NO}]^{-}$} & 5/n.d. & 193 & n.d. \\
\hline 1,6-Dinitropyrene & $\mathrm{C}_{16} \mathrm{H}_{8} \mathrm{~N}_{2} \mathrm{O}_{4}$ & 35.9 & {$\left[\mathbf{M}^{\cdot}\right]^{-},[\mathbf{M}-\mathbf{N O}]^{-}$} & $25 /$ n.d. & 262 & n.d. \\
\hline 1,8-Dinitropyrene & $\mathrm{C}_{16} \mathrm{H}_{8} \mathrm{~N}_{2} \mathrm{O}_{4}$ & 37.3 & {$\left[\mathbf{M}^{-}\right]^{-},\left[\mathbf{M}-\mathbf{N O}^{-}\right]^{-}$} & $25 /$ n.d. & 262,232 & n.d. \\
\hline 4-Nitropyrene & $\mathrm{C}_{16} \mathrm{H}_{9} \mathrm{NO}_{2}$ & 37.3 & {$\left[\mathrm{M}^{-}\right]^{-},[\mathrm{M}-\mathrm{NO}]^{-}$} & 5/n.d. & 217,232 & n.d. \\
\hline 1-Nitropyrene & $\mathrm{C}_{16} \mathrm{H}_{9} \mathrm{NO}_{2}$ & 37.4 & {$\left[\mathrm{M}^{-}\right]^{-},\left[\mathrm{M}-\mathrm{NO}^{-}\right]^{-},[\mathrm{M}+\mathrm{H}]^{+}$} & 5/n.d. & 217 & $231,202,248,218$ \\
\hline 3-Nitrofluoranthene & $\mathrm{C}_{16} \mathrm{H}_{9} \mathrm{NO}_{2}$ & 37.5 & {$\left[\mathrm{M}^{-}\right]^{-},\left[\mathrm{M}-\mathrm{NO}^{-}\right]^{-}$} & 25/n.d. & 217 & n.d. \\
\hline 1,3-Dinitropyrene & $\mathrm{C}_{16} \mathrm{H}_{8} \mathrm{~N}_{2} \mathrm{O}_{4}$ & 37.9 & {$\left[\mathbf{M}^{-}\right]^{-},\left[\mathbf{M}-\mathbf{N O}^{-}\right]^{-}$} & $25 / 500$ & 262,232 & n.d. \\
\hline 2-Nitropyrene & $\mathrm{C}_{16} \mathrm{H}_{9} \mathrm{NO}_{2}$ & 38.7 & {$\left[\mathbf{M}^{\cdot}\right]^{-},\left[\mathrm{M}-\mathrm{NO}^{*}\right]^{-}$} & $25 / 50$ & 217 & n.d. \\
\hline 7-Nitrobenz[a]anthracene & $\mathrm{C}_{18} \mathrm{H}_{11} \mathrm{NO}_{2}$ & 39.0 & {$\left[\mathbf{M}^{-}\right]^{-},\left[\mathrm{M}-\mathrm{NO}^{\cdot}\right]^{-}$} & 25/n.d. & 243 & n.d. \\
\hline 6-Nitrochrysene & $\mathrm{C}_{18} \mathrm{H}_{11} \mathrm{NO}_{2}$ & 40.0 & {$\left[\mathbf{M}^{-}\right]^{-},\left[\mathrm{M}-\mathrm{NO}^{-}\right]^{-},[\mathrm{M}+\mathrm{H}]^{+}$} & 5/n.d. & 243 & $274,257,244,228$ \\
\hline 6-Nitrobenzo[a]pyrene & $\mathrm{C}_{20} \mathrm{H}_{11} \mathrm{NO}_{2}$ & 42.6 & {$\left[\mathbf{M}^{-}\right]^{-},\left[\mathrm{M}-\mathrm{NO}^{\cdot}\right]^{-},[\mathrm{M}+\mathrm{H}]^{+}$} & $5 / 25$ & 267 & $281,298,267,252$ \\
\hline \multicolumn{7}{|l|}{ Keto-PAHs } \\
\hline 9-Fluorenone & $\mathrm{C}_{13} \mathrm{H}_{8} \mathrm{O}$ & 25.3 & {$[\mathbf{M}+\mathbf{H}]^{+},\left[\mathrm{M}^{\cdot}\right]^{-},[\mathrm{M}-\mathrm{H}]^{-}$} & $500 / 50$ & $180,165,162,152,150$ & 181, 153, 152 \\
\hline 4H-Cyclopenta[def]phenanthren-4-one & $\mathrm{C}_{15} \mathrm{H}_{8} \mathrm{O}$ & 30.5 & {$\left[\mathbf{M}^{-}\right]^{-},[\mathrm{M}+\mathrm{H}]^{+}$} & $25 / 50$ & 204, 174, 189 & $205,177,176$ \\
\hline Cyclopenta[cd]pyrene-3[4H]-one & $\mathrm{C}_{18} \mathrm{H}_{10} \mathrm{O}$ & 33.4 & {$[\mathbf{M}-\mathbf{H}]^{-},[\mathrm{M}+\mathrm{H}]^{+}$} & $5 / 25$ & $241,211,223,225$ & $243,215,226,225,201,242$ \\
\hline $6 \mathrm{H}$-Benzo[cd]pyrene-6-one & $\mathrm{C}_{19} \mathrm{H}_{10} \mathrm{O}$ & 37.1 & {$\left[\mathbf{M}^{\prime}\right]^{-},[\mathrm{M}+\mathrm{H}]^{+}$} & $25 / 25$ & $254,224,236$ & $255,227,226$ \\
\hline \multicolumn{7}{|l|}{ Nitro-keto-PAHs } \\
\hline 2-Nitrofluorenon & $\mathrm{C}_{13} \mathrm{H}_{7} \mathrm{NO}_{3}$ & 26.9 & {$\left[\mathbf{M}^{-}\right]^{-},[\mathrm{M}+\mathrm{H}]^{+}$} & $25 /$ n.d. & 195, 197 & 209, 180, 207, 198 \\
\hline 3-Nitrobenzanthrone & $\mathrm{C}_{17} \mathrm{H}_{9} \mathrm{NO}_{3}$ & 33.9 & {$\left[\mathbf{M}^{-}\right]^{-},[\mathrm{M}+\mathrm{H}]^{+}$} & $5 / 50$ & 245,247 & $259,246,230,218$ \\
\hline \multicolumn{7}{|l|}{$\mathrm{OH}-\mathrm{PAHs}$} \\
\hline 1-Naphthol & $\mathrm{C}_{10} \mathrm{H}_{8} \mathrm{O}$ & 22.8 & {$[\mathrm{M}-\mathrm{H}]^{-},[\mathrm{M}+\mathrm{H}]^{+},[\mathrm{M}]^{+}$} & 25/n.d. & $143,128,115$ & $145,117,127$ \\
\hline 9-Phenanthrol & $\mathrm{C}_{14} \mathrm{H}_{10} \mathrm{O}$ & 31.9 & {$[\mathbf{M}-\mathbf{H}]^{-},[\mathrm{M}+\mathrm{H}]^{+},[\mathrm{M}]^{+}$} & 50/n.d. & $193,165,175,176$ & 195,167 \\
\hline 1-Hydroxypyrene & $\mathrm{C}_{16} \mathrm{H}_{10} \mathrm{O}$ & 37.2 & {$[\mathbf{M}-\mathbf{H}]^{-},[\mathrm{M}+\mathrm{H}]^{+},[\mathrm{M}]^{+}$} & $25 / 250$ & 217, 199, 189 & $219,201,191,218,202,203$ \\
\hline 3-Hydroxybenzo[a]pyrene & $\mathrm{C}_{20} \mathrm{H}_{12} \mathrm{O}$ & 45.0 & {$[\mathbf{M}-\mathbf{H}]^{-},[\mathrm{M}+\mathrm{H}]^{+},[\mathrm{M}]^{+}$} & 5/n.d. & $267,252,249,239$ & $269,251,252,268$ \\
\hline \multicolumn{7}{|l|}{ Quinone } \\
\hline 1,4-Naphthoquinone & $\mathrm{C}_{10} \mathrm{H}_{6} \mathrm{O}_{2}$ & 11.6 & {$\left[\mathbf{M}^{-}\right]^{-},[\mathrm{M}+\mathrm{H}]^{+}$} & $25 / 250$ & 158,130 & $159,131,103$ \\
\hline 1,2-Acenaphthenequinone & $\mathrm{C}_{12} \mathrm{H}_{6} \mathrm{O}_{2}$ & 12.4 & {$\left[\mathbf{M}^{-}\right]^{-},[\mathrm{M}+\mathrm{H}]^{+}$} & $25 /$ n.d. & $182,154,165,141$ & 183, 155, 145, 165, 127, 105 \\
\hline 9,10-Phenanthrenedione & $\mathrm{C}_{14} \mathrm{H}_{8} \mathrm{O}_{2}$ & 19.6 & {$\left[\mathbf{M}^{-}\right]^{-},[\mathrm{M}+\mathrm{H}]^{+}$} & $5 / 25$ & 208, 180, 164, 193 & 209, 181, 153 \\
\hline 1,6-Pyrenequinone & $\mathrm{C}_{16} \mathrm{H}_{8} \mathrm{O}_{2}$ & 19.9 & {$\left[\mathbf{M}^{-}\right]^{-},[\mathrm{M}+\mathrm{H}]^{+}$} & $5 / 25$ & 232, 214, 204 & 233, 205, 177 \\
\hline 1,4-Anthraquinone & $\mathrm{C}_{14} \mathrm{H}_{8} \mathrm{O}_{2}$ & 23.5 & {$\left[\mathbf{M}^{-}\right]^{-},[\mathrm{M}+\mathrm{H}]^{+}$} & $5 / 25$ & $208,180,164,193$ & 209, 181, 153 \\
\hline 9,10-Anthraquinone & $\mathrm{C}_{14} \mathrm{H}_{8} \mathrm{O}_{2}$ & 27.0 & {$\left[\mathbf{M}^{-}\right]^{-},[\mathrm{M}+\mathrm{H}]^{+}$} & $25 / 50$ & $208,180,164,193$ & 209, 181, 153 \\
\hline 2-Methyl anthraquinone & $\mathrm{C}_{15} \mathrm{H}_{10} \mathrm{O}_{2}$ & 30.6 & {$\left[\mathbf{M}^{-}\right]^{-},[\mathrm{M}-\mathrm{H}]^{-},[\mathrm{M}+\mathrm{H}]^{+}$} & $25 / 250$ & $222,194,178,131,207$ & $\begin{array}{l}\text { 223, 195, 177, 167, 205, 152, 221, 166, 165, } \\
\quad 178,179,193,194\end{array}$ \\
\hline Benz[a]anthracene-7,12-dione & $\mathrm{C}_{18} \mathrm{H}_{10} \mathrm{O}_{2}$ & 38.3 & {$\left[\mathbf{M}^{-}\right]^{-},[\mathrm{M}+\mathrm{H}]^{+}$} & $5 / 250$ & $258,240,230,214,243$ & $259,231,215,203,241,240,202$ \\
\hline Anthanthrone & $\mathrm{C}_{22} \mathrm{H}_{10} \mathrm{O}_{2}$ & 42.4 & {$\left[\mathbf{M}^{-}\right]^{-},[\mathrm{M}+\mathrm{H}]^{+}$} & $5 / 25$ & $306,288,278,274$ & $307,279,251,306,250,278$ \\
\hline \multicolumn{7}{|l|}{$\mathrm{OH}-Q u i n o n e$} \\
\hline 2-Hydroxyanthraquinone & $\mathrm{C}_{14} \mathrm{H}_{8} \mathrm{O}_{3}$ & 27.4 & {$[\mathbf{M}-\mathbf{H}]^{-},[\mathrm{M}+\mathrm{H}]^{+}$} & $25 / 25$ & 223,195 & $225,197,169,183,153,207,105,141,181,171$ \\
\hline 1-Hydroxyanthraquinone & $\mathrm{C}_{14} \mathrm{H}_{8} \mathrm{O}_{3}$ & 31.3 & {$\left[\mathbf{M}^{-}\right]^{-},[\mathrm{M}-\mathrm{H}]^{-},[\mathrm{M}+\mathrm{H}]^{+}$} & $25 / 250$ & 224, 196, 195 & $225,197,207,183,169,153,105,141,181,171$ \\
\hline 1,3-Dihydroxyanthraquinone & $\mathrm{C}_{14} \mathrm{H}_{8} \mathrm{O}_{4}$ & 32.2 & {$[\mathrm{M}-\mathrm{H}]^{-},[\mathrm{M}+\mathrm{H}]^{+}$} & $25 / 50$ & 239, 211, 195 & $199,171,223,213$ \\
\hline 1,8-Dihydroxyanthraquinone & $\mathrm{C}_{14} \mathrm{H}_{8} \mathrm{O}_{4}$ & 33.6 & {$\left[\mathbf{M}^{-}\right]^{-},[\mathrm{M}-\mathrm{H}]^{-},[\mathrm{M}+\mathrm{H}]^{+}$} & $25 /$ n.d. & 240,212 & $\begin{array}{l}\text { 241, 213, 121, 223, 200, 185, 169, 141, 157, } \\
\quad 197,195,167\end{array}$ \\
\hline
\end{tabular}

37.9

$\mathrm{C}_{18} \mathrm{H}_{11} \mathrm{NO}_{2} \quad 39.0$

40.0

42.6

30.5

4H-Cyclopentaldef]phenanthren-4-one

$6 \mathrm{H}$-Benzo[ $[c d]$ pyrene-6-one

$\mathrm{C}_{19} \mathrm{H}_{10} \mathrm{O}$

33.4

$\left[\mathrm{M}^{\cdot}\right]^{-},[\mathrm{M}+\mathrm{H}]^{+}$

itro-keto-PAHs

3-Nitrobenzanthrone

$\mathrm{C}_{17} \mathrm{H}_{9} \mathrm{NO}_{3} \quad 33.9 \quad\left[\mathrm{M}^{-}\right]^{-},[\mathrm{M}+\mathrm{H}]^{+}$

$\mathrm{C}_{10} \mathrm{H}_{8} \mathrm{O}$

31.9

M-H $]^{-},[\mathrm{M}+\mathrm{H}]^{+},[\mathrm{M}]^{+}$

(M-H $]^{-},[\mathrm{M}+\mathrm{H}]^{+},[\mathrm{M}]^{+}$

1-Hydroxypyren

$\mathrm{C}_{20} \mathrm{H}_{12} \mathrm{O}$

$[\mathbf{M}-\mathbf{H}]^{-},[\mathrm{M}+\mathrm{H}]^{+},[\mathrm{M}]^{+}$

193, $165,175,17$

$17,199,189$

$219,201,191,218,202,203$

Quinone

1,4-Naphthoquinone

10-Phenanthrenedione

$\mathrm{C}_{14} \mathrm{H}_{8} \mathrm{O}_{2}$

$\mathrm{C}_{14} \mathrm{H}_{8} \mathrm{O}_{2}$

$\left[\mathbf{M}^{-}\right]^{-},[\mathrm{M}+\mathrm{H}]$

$[\mathrm{M}]^{-},[\mathrm{M}+\mathrm{H}]^{+}$

$\mathrm{C}_{15} \mathrm{H}_{10} \mathrm{O}$

$\left.\mathbf{M}^{-}\right]^{-},[\mathrm{M}-\mathrm{H}]^{-},[\mathrm{M}+\mathrm{H}]^{+}$ 
Table 1. Continued

\begin{tabular}{|c|c|c|c|c|c|c|}
\hline & EF & $\begin{array}{c}\mathrm{Rt} \\
{[\mathrm{min}]}\end{array}$ & Observed signal & $\mathrm{DL}(\mathrm{pg})$ & $\begin{array}{c}\text { MS/MS } \\
\text { (negative mode) }\end{array}$ & $\begin{array}{c}\text { MS/MS } \\
\text { (positive mode) }\end{array}$ \\
\hline \multicolumn{7}{|l|}{ Amino-PAHs } \\
\hline 2-Aminoanthracene & $\mathrm{C}_{14} \mathrm{H}_{11} \mathrm{~N}$ & 29.5 & {$[\mathrm{M}+\mathrm{H}]^{+},[\mathrm{M}-\mathrm{H}]^{-}$} & $100 / 50$ & 192, 165, 177 & 194, 177, 178, 167, 179, 176, 166, 165, 192, 193 \\
\hline 4-Aminopyrene & $\mathrm{C}_{16} \mathrm{H}_{11} \mathrm{~N}$ & 32.0 & {$[\mathrm{M}+\mathrm{H}]^{+},[\mathrm{M}-\mathrm{H}]^{-}$} & $50 / 25$ & 216, 188, 201 & $218,202,201,217,191,216,200$ \\
\hline 1-Aminopyrene & $\mathrm{C}_{16} \mathrm{H}_{11} \mathrm{~N}$ & 32.6 & {$[\mathbf{M}+\mathbf{H}]^{+},[\mathrm{M}-\mathrm{H}]^{-}$} & $50 / 25$ & 216,188 & $218,202,201,217,190,216,200,191,189$ \\
\hline 3-Aminofluoranthene & $\mathrm{C}_{16} \mathrm{H}_{11} \mathrm{~N}$ & 32.6 & {$[\mathrm{M}+\mathrm{H}]^{+},[\mathrm{M}-\mathrm{H}]^{-}$} & $25 / 25$ & $216,188,165$ & $218,191,202,217,201$ \\
\hline 2-Aminopyrene & $\mathrm{C}_{16} \mathrm{H}_{11} \mathrm{~N}$ & 33.4 & {$[\mathrm{M}+\mathrm{H}]^{+},[\mathrm{M}-\mathrm{H}]^{-}$} & $50 / 25$ & 216,188 & $218,202,201,217$ \\
\hline 1,3-Diaminopyrene & $\mathrm{C}_{16} \mathrm{H}_{12} \mathrm{~N}_{2}$ & 35.9 & {$[\mathbf{M}+\mathbf{H}]^{+},[\mathrm{M}-\mathrm{H}]^{-}$} & n.d./25 & 231, 201 & $233,217,205,232,202,203,206,216,231,218$ \\
\hline 1,8-Diaminopyrene & $\mathrm{C}_{16} \mathrm{H}_{12} \mathrm{~N}_{2}$ & 37.3 & {$[\mathrm{M}+\mathrm{H}]^{+},[\mathrm{M}-\mathrm{H}]^{-}$} & $50 / 25$ & 231, 201 & $233,217,216,232,189,205,203,231$ \\
\hline 1,6-Diaminopyrene & $\mathrm{C}_{16} \mathrm{H}_{12} \mathrm{~N}_{2}$ & 37.8 & {$[\mathrm{M}+\mathrm{H}]^{+},[\mathrm{M}-\mathrm{H}]^{-}$} & $50 / 25$ & 231,201 & 233, 217, 216, 232, 204, 189, 204, 205 \\
\hline 6-Aminochrysene & $\mathrm{C}_{1} \mathrm{H}_{13} \mathrm{~N}$ & 35.4 & {$[\mathbf{M}+\mathbf{H}]^{+},[\mathrm{M}-\mathrm{H}]^{-}$} & $250 / 50$ & $242,240,215,221$ & $\begin{array}{l}\mathbf{2 4 4}, \mathbf{2 1 7}, \mathbf{2 2 7}, \mathbf{2 2 8}, \mathbf{2 4 3}, 216,215,226,229 \\
\quad 242,241,203,189\end{array}$ \\
\hline 6-Aminobenzo[a]pyrene & $\mathrm{C}_{20} \mathrm{H}_{13} \mathrm{~N}$ & 41.0 & {$[\mathrm{M}+\mathrm{H}]^{+},[\mathrm{M}-\mathrm{H}]^{-}$} & $5 / 25$ & $266,239,251$ & $267,252,267,266,251,241$ \\
\hline \multicolumn{7}{|l|}{ Azzaarene } \\
\hline Carbazole & $\mathrm{C}_{12} \mathrm{H}_{9} \mathrm{~N}$ & 29.2 & {$[\mathbf{M}+\mathbf{H}]^{+},[\mathrm{M}-\mathrm{H}]^{-}$} & $25 / 50$ & $166,150,136,122$ & $168,141,139$ \\
\hline N-Phenyl-2-naphthylamine & $\mathrm{C}_{16} \mathrm{H}_{13} \mathrm{~N}$ & 33.8 & {$[\mathrm{M}+\mathrm{H}]^{+},[\mathrm{M}-\mathrm{H}]^{-}$} & $25 / 25$ & 218,216 & $\begin{array}{l}\text { 143, 205, 203, 142, 92, 193, 115, 128, 218, 217, } \\
\quad 178,191\end{array}$ \\
\hline Phenanthridine & $\mathrm{C}_{13} \mathrm{H}_{9} \mathrm{~N}$ & 23.0 & {$[\mathbf{M}+\mathbf{H}]^{+}$} & n.d./25 & n.d. & $180,165,153,152,151$ \\
\hline Acridine & $\mathrm{C}_{13} \mathrm{H}_{9} \mathrm{~N}$ & 23.1 & {$[\mathbf{M}+\mathbf{H}]^{+}$} & n.d./25 & n.d. & 180,165 \\
\hline Benzo $[h]$ quinoline & $\mathrm{C}_{13} \mathrm{H}_{9} \mathrm{~N}$ & 25.3 & {$[\mathbf{M}+\mathbf{H}]^{+}$} & n.d./25 & n.d. & $180,152,150$ \\
\hline Benz[a]acridine & $\mathrm{C}_{17} \mathrm{H}_{11} \mathrm{~N}$ & 31.6 & {$[\mathbf{M}+\mathbf{H}]^{+}$} & n.d. $/ 25$ & n.d. & $230,203,202$ \\
\hline Benz $[c]$ acridine & $\mathrm{C}_{17} \mathrm{H}_{11} \mathrm{~N}$ & 36.3 & {$[\mathbf{M}+\mathbf{H}]^{+}$} & n.d./50 & n.d. & $230,202,203,201,213$ \\
\hline 10-Azabenzo[a]pyrene & $\mathrm{C}_{19} \mathrm{H}_{11} \mathrm{~N}$ & 39.1 & {$[\mathbf{M}+\mathbf{H}]^{+}$} & n.d./25 & n.d. & $254,226,253$ \\
\hline Dibenz $[a, j]$ acridine & $\mathrm{C}_{21} \mathrm{H}_{13} \mathrm{~N}$ & 38.8 & {$[\mathbf{M}+\mathbf{H}]^{+}$} & n.d. $/ 25$ & n.d. & $280,278,253$ \\
\hline
\end{tabular}

The tandem mass spectral signals of highest relative intensities are in bold font.

aDetection limit in negative ion mode.

Detection limit in positive ion mode.

n.d. $=$ not detected. 


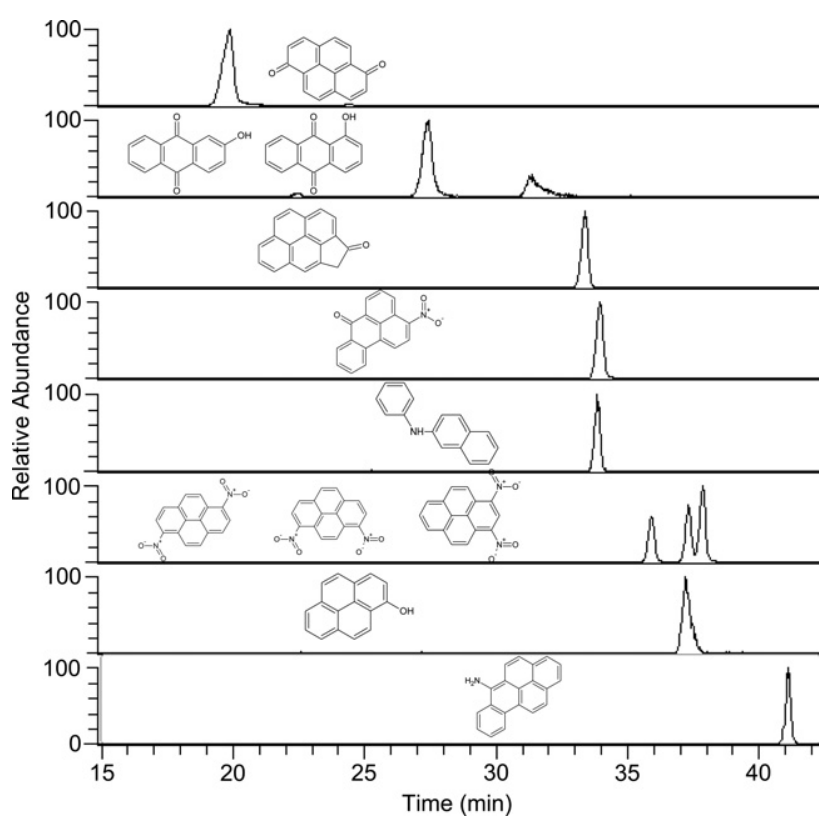

Figure 1. RP-HPLC/APCI-HR-MS extracted ion chromatograms of some selected model compounds.

than seven condensed rings covering a wide range of estimated octanol-water partition coefficients ( Log P 1.8-5.9) representing nitro-PAHs, keto-PAHs, nitro-ketoPAHs, hydroxy-PAHs, quinones, hydroxyl-quinones, amino-PAHs, and azaarenes as listed in Table 1. The selected partial polar PACs were separated on a polymeric RP-C18 column and detected by APCI-MS. To elucidate unknown PACs in mutagenic fractions, understanding the chromatographic behavior, the ion formation in the APCI source including in-source fragmentation, and tandem MS/MS fragmentation in the linear ion trap is crucial. For the model compounds the exact masses, chromatographic retention times, physical parameters, limits of detections, mass spectrometric signals observed in positive and negative APCI ion mode, and MS fragmentation data are listed in Table 1 and Table S-1.

\section{Behavior of Model Compounds}

Chromatography of model compounds. All selected model substances, except azaarenes and amino-PAHs, are not ionized in solution. The peak shapes for pure standard mixture on polymeric RP-C18 for all compounds were characterized by some fronting but no tailing (Figure 1). In order not to reduce ionization efficiency in negative ion mode, no mobile phase modifier (e.g., formic acid) was used. The polymeric stationary phase is expected to separate complex mixtures of PACs including isomers based on hydrophobicity expressed as $\log \mathrm{P}$, intramolecular steric hindrance due to bay positions and peri hydrogens, and maximum length to breadth (L/B) ratios [39]. It was observed within one class of compounds, retention times exhibited a regular increase with molecular mass $(\log P)$, and a systematic decrease with increasing number of polar functional groups (Figure 2a). 1-Nitronaphthalene (two fused rings) elutes at Rt $24.6 \mathrm{~min}$ and a higher number of fused rings increases the retention as for 1-nitropyrene (four fused rings; Rt $37.4 \mathrm{~min}$ ) and 6-nitrobenzo[a]pyrene (five fused rings; $42.6 \mathrm{~min}$ ). The separation trend is very similar as characterized by Letzel et al. on a phenylmodified reversed-phase column for some similar compound classes [40]. Retention of structural isomers was most affected by the presence of substituents in bay position, but also by the number of $\mathrm{H}$ atoms in peri position to the substituent group and by the L/B ratio of the molecule (Figure $2 \mathrm{~b}$ ). The presence of a bay region in benzo[ $h]$ quinoline resulted in a longer retention time (Rt $25.3 \mathrm{~min}$ ) compared to the two isomers acridine (Rt. $23.1 \mathrm{~min} ; 2$ peri hydrogens), and phenanthridine (Rt 23.0 min, 1 peri hydrogen). An isomer with 2 peri hydrogens as in 1,4-anthraquinone eluted at $11.6 \mathrm{~min}$ before 9,10anthraquinone (Rt 27.0), with four peri hydrogens. The same phenomena have been observed for 1- and 2hydroxyanthraquinone, 1,3-and 1,8-dihydroxyanthraquinone, and benzo[ $[a]$ acridine and benzo[c]acridine isomers. The retention times for isomers with similar Log $\mathrm{P}$ and the absence of intramolecular steric hindrance were longer for greater L/B ratios, e.g., as for 1-, 2-, and
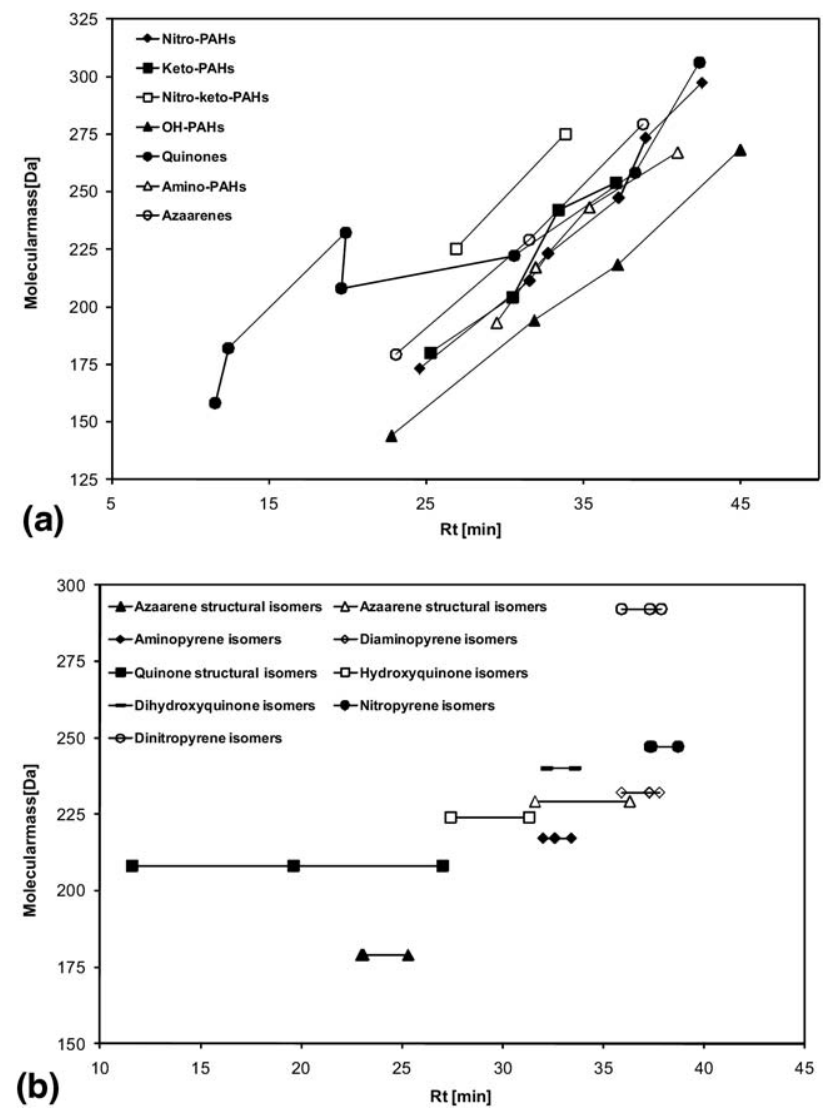

Figure 2. (a) Chromatographic retention behavior of the nonstructural isomers of the selected model compounds on polymeric RP-C18 column; (b) structural isomers behavior of the selected model compounds on polymeric RP-C18. 
Table 2. Summary of MS classifiers for selected classes based on ion formation during APCI ionization including in-source fragmentation, and tandem MS/MS fragmentation

\begin{tabular}{|c|c|c|c|}
\hline Model class & Observed signals & Diagnostic fragments APCI (-) & Diagnostic fragments APCl $(+)$ \\
\hline Nitro-PAHs & $\begin{array}{l}{ }^{\mathrm{a}}\left[\mathbf{M}^{\cdot}\right]^{-},{ }^{\mathrm{b}}[\mathbf{M}-\mathbf{H}]^{-},[\mathrm{M}-\mathrm{NO}]^{-}, \\
{ }^{\mathrm{C}}[\mathrm{M}+\mathrm{H}]^{+}\end{array}$ & {$[\mathrm{M}-\mathrm{NO}]^{-}, \mathrm{h}[\mathrm{M}-2 \mathrm{NO}]^{-}$} & {$[\mathrm{M}+\mathrm{H}-\mathrm{OH}]^{+},\left[\mathrm{M}+\mathrm{H}-\mathrm{NO}_{2}\right]^{+},[\mathrm{M}+\mathrm{H}-\mathrm{NO}]^{+}$} \\
\hline Nitroketo-PAHs & {$[\mathbf{M}]^{-},[\mathrm{M}+\mathrm{H}]^{+}$} & {$[\mathrm{M}-\mathrm{NO}]^{-},[\mathrm{M}-\mathrm{CO}]^{-}$} & {$[\mathrm{M}+\mathrm{H}-\mathrm{OH}]^{+},[\mathrm{M}+\mathrm{H}-\mathrm{NO}]^{+},\left[\mathrm{M}+\mathrm{H}-\mathrm{NO}_{2}\right]^{+}$} \\
\hline Keto-PAHs & ${ }^{\mathrm{a}}[\mathbf{M}]^{-},{ }^{\mathrm{b}}[\mathbf{M}-\mathbf{H}]^{-},[\mathrm{M}+\mathrm{H}]^{+}$ & $\begin{array}{l}{\left[\mathrm{M}-\mathrm{H}_{2}-\mathrm{CO}\right]^{-},\left[\mathrm{M}-\mathrm{H}_{2} \mathrm{O}\right]^{-}} \\
{\left[\mathrm{M}-\mathrm{CH}_{3}\right]^{-}}\end{array}$ & {$[\mathrm{M}+\mathrm{H}-\mathbf{C O}]^{+},{ }^{\mathrm{a}}[\mathrm{M}+\mathrm{H}-\mathrm{HCO}]^{+}$} \\
\hline $\mathrm{OH}-\mathrm{PAHs}$ & {$[\mathrm{M}-\mathrm{H}]^{-},[\mathrm{M}+\mathrm{H}]^{+},[\mathrm{M} \cdot]^{+}$} & {$[\mathrm{M}-\mathrm{H}-\mathrm{CO}]^{-},\left[\mathrm{M}-\mathrm{H}-\mathrm{H}_{2} \mathrm{O}\right]^{-}$} & $\begin{array}{l}{[\mathbf{M}+\mathbf{H}-\mathbf{C O}]^{+},{ }^{j}\left[\mathbf{M}+\mathbf{H}-\mathbf{H}_{2} \mathbf{O}\right]^{+},{ }^{j}[\mathbf{M}+\mathbf{H}-\mathbf{H}]^{+}} \\
\quad[\mathrm{M}+\mathrm{H}-\mathrm{OH}]^{+}\end{array}$ \\
\hline Quinones & {$\left[\mathbf{M}^{-}\right]^{-},{ }^{\mathrm{d}}[\mathrm{M}-\mathrm{H}]^{-},[\mathrm{M}+\mathrm{H}]^{+}$} & $\begin{array}{l}{[\mathrm{M}-\mathrm{CO}]^{-},\left[\mathrm{M}-\mathrm{CO}_{2}\right]^{-},\left[\mathrm{M}-\mathrm{H}_{2} \mathrm{O}\right]^{-},} \\
\quad\left[\mathrm{M}-\mathrm{CH}_{3}\right]^{-}\end{array}$ & $\begin{array}{l}{[\mathrm{M}+\mathrm{H}-\mathrm{CO}]^{+},[\mathrm{M}+\mathrm{H}-2 \mathrm{CO}]^{+},\left[\mathrm{M}+\mathrm{H}-\mathrm{H}_{2} \mathrm{O}\right]^{+},} \\
{ }^{\mathrm{e}}\left[\mathrm{M}+\mathrm{H}-\mathrm{H}_{2}\right]^{+}, \mathrm{e}[\mathrm{M}+\mathrm{H}-\mathrm{HCO}]^{+}, \\
{ }^{\mathrm{e}}[\mathrm{M}+\mathrm{H}-2 \mathrm{HCO}]^{+}, \mathrm{e}\left[\mathrm{M}+\mathrm{H}-\mathrm{CO}_{2}\right]^{+}, \\
{ }^{\mathrm{e}}\left[\mathrm{M}+\mathrm{H}-\mathrm{HCO}_{2}\right]^{+},{ }^{\mathrm{f}}\left[\mathrm{M}+\mathrm{H}-\mathrm{CO}_{2}\right]^{+}, \\
{ }^{\mathrm{f}}[\mathrm{M}+\mathrm{H}-\mathrm{OH}]^{+}\end{array}$ \\
\hline OH-Quinones & $\begin{array}{l}{[\mathbf{M}-\mathbf{H}]^{-},{ }^{g}[\mathbf{M}]^{-},{ }^{9}[\mathrm{M}-\mathrm{H}]^{-}} \\
\quad[\mathrm{M}+\mathrm{H}]^{+}\end{array}$ & $\begin{array}{l}{[\mathrm{M}-\mathrm{H}-\mathbf{C O}]^{-}, \mathrm{g}[\mathrm{M}-\mathrm{CO}]^{-}} \\
\quad \mathrm{g}[\mathrm{M}-\mathrm{HCO}]^{-}\end{array}$ & $\begin{array}{l}{[\mathrm{M}+\mathrm{H}-\mathrm{CO}]^{+},[\mathrm{M}+\mathrm{H}-\mathbf{2 C O}]^{+}} \\
\quad\left[\mathrm{M}+\mathrm{H}-\mathrm{CO}_{2}-\mathrm{CO}\right]^{+},\left[\mathrm{M}+\mathrm{H}-\mathrm{H}_{2} \mathrm{O}\right]^{+} \\
{\left[\mathrm{M}+\mathrm{H}-\mathrm{CO}_{2}\right]^{+},[\mathrm{M}+\mathrm{H}-3 \mathrm{CO}]^{+}}\end{array}$ \\
\hline Amino-PAHs & {$[\mathbf{M}+\mathbf{H}]^{+},[\mathrm{M}-\mathrm{H}]^{-}$} & $\begin{array}{l}{ }^{\mathrm{k}}[\mathrm{M}-\mathrm{H}-\mathrm{HCN}]^{-},{ }^{\mathrm{k}}\left[\mathrm{M}-\mathrm{H}-\mathrm{H}_{2} \mathrm{CN}\right]^{-}, \\
{ }^{\mathrm{i}}\left[\mathrm{M}-\mathrm{H}-\mathrm{H}_{2} \mathrm{CN}-\mathrm{H}_{2}\right]^{-}, \\
{[\mathrm{M}-\mathrm{H}-\mathrm{NH}]^{-}}\end{array}$ & 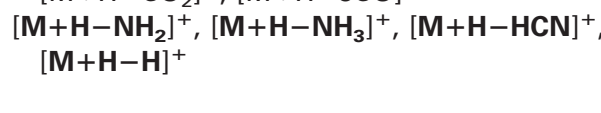 \\
\hline Azaarenes & {$[\mathbf{M}+\mathbf{H}]^{+}$} & & {$[\mathrm{M}+\mathrm{H}-\mathrm{HCN}]^{+},\left[\mathrm{M}+\mathrm{H}-\mathrm{H}_{\mathbf{2}} \mathrm{CN}\right]^{+},[\mathrm{M}+\mathrm{H}-\mathrm{NH}]^{+}$} \\
\hline
\end{tabular}

The bold font indicates high relative intensity.

aFull aromatic structure.

bPartly aromatic structure.

"Some molecules from the class were observed.

dMethylated structure.

eAdditional fragments observed in methylated quinone.

fAdditional fragments observed in quinone structure with bay region.

gHydroxy-quinones with intramolecular interaction.

hDinitro-PAHs.

iDiamino-PAHs.

intensity increases with increasing molecular weights.

${ }^{\mathrm{k}}$ mono-amino-PAHs.

4-aminopyrene and for 1-, 2-, and 4-nitropyrene (Table $\mathrm{S}-1$, Supporting Information; structural information is shown).

Ionization of model compounds. Since most of the model compounds are not ionizable in solution, APCI was chosen as ionization technique instead of electrospray ionization, and the negative and the positive ion mode were applied (Table 1). For some molecules both ion modes were suitable. As outlined in Table 2, molecules without acidic hydrogens (nitro-PAHs, keto-PAHs, nitro-keto-PAHs, and quinones) were observed in the positive mode as protonated ion $[\mathrm{M}+\mathrm{H}]^{+}$and in the negative mode as radical anions $[\mathrm{M} \cdot]^{-}$. However, in the presence of partially aromatic ring structures formation of deprotonated molecules $[\mathrm{M}-\mathrm{H}]^{-}$is also observed. Molecules with acidic hydrogens and no intramolecular H-bonds (hydroxyl-PAHs and hydroxylquinones) were observed as $[\mathrm{M}+\mathrm{H}]^{+}$in the positive mode, whereas $[\mathrm{M}-\mathrm{H}]^{-}$was detected in the negative mode. In the case of intramolecular H-bonds radical anions instead of deprotonated molecules were observed. Basic molecules like azaarenes and aminoPAHs were observed in the positive ion mode as [M + $\mathrm{H}]^{+}$. Amino-PAHs were also observed as $[\mathrm{M}-\mathrm{H}]^{-}$in the negative ion mode. The detection limits in full scan MS were compound class-dependent and in the optimal ionization mode polarity the detection limits were below $25 \mathrm{pg}$ on column as shown in Table 1 .

\section{Tandem MS Product Ion Interpretation}

The use of the hybrid LIT-FTICR-MS not only provides high-resolution MS but also high mass accuracy with less than 1 ppm error as shown in Table S-1. So, this is

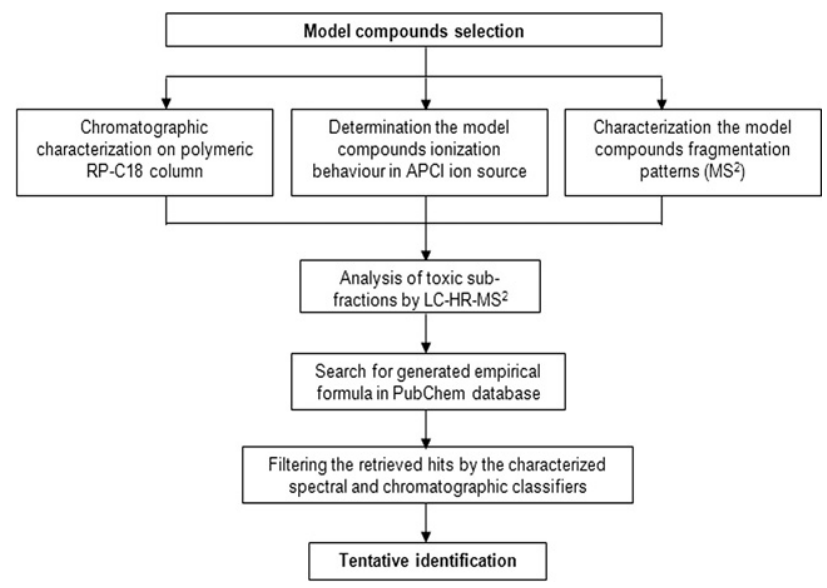

Figure 3. Tentative identification approach based on PubChem database and its integration with characterized classifiers by using model compounds containing mutagenic substructures. 

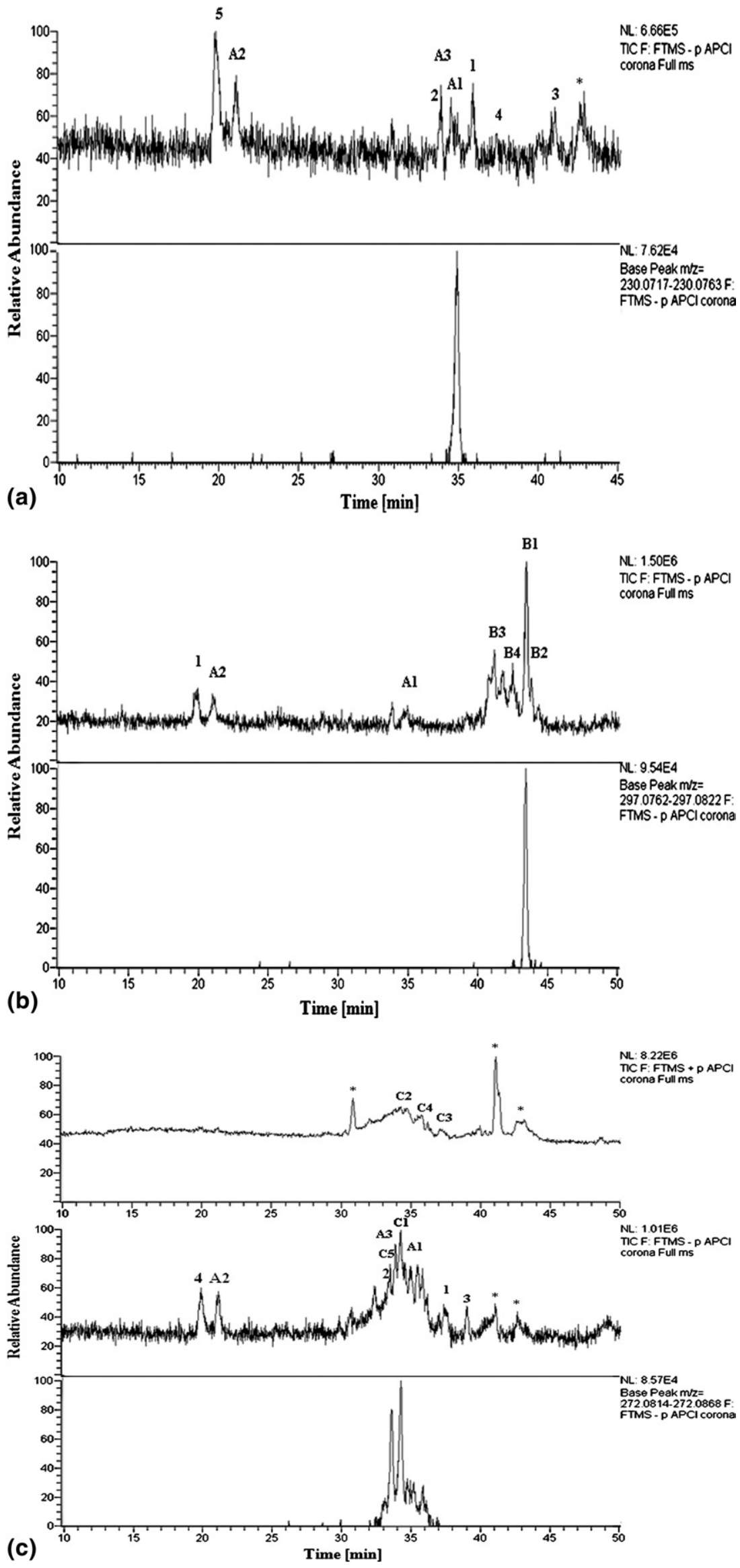
enabling the unambiguous determination of elemental compositions and the determination of exact masses of fragment ions in tandem MS/MS. The results of collision induced dissociation experiments are summarized in Table 2.

Nitro-PAHs were detected in negative mode as radical anions $[\mathrm{M} \cdot]^{-}[41]$, and significant intensities of [M $\mathrm{NO}^{-}$[41] were observed. The latter signals were raised with increasing molecular weights. Interestingly, 2nitrofluorene was detected as deprotonated molecule $[\mathrm{M}-\mathrm{H}]^{-}$. This may be due to the presence of a partially hydrogenated ring system. In negative ion mode the tandem MS for all nitro-PAHs showed a diagnostic fragment ion indicating the neutral loss of NO [41]. Since other PACs do not undergo a similar fragmentation, this difference can be exploited for selective identification of nitro-PAHs in complex environmental mixtures by using constant neutral loss scan. A few nitroPAHs were detected in positive ion mode as $[\mathrm{M}+\mathrm{H}]^{+}$, and the tandem MS showed the main fragment ions as $\left[\mathrm{M}+\mathrm{H}-\mathrm{NO}_{2}\right]^{+},[\mathrm{M}+\mathrm{H}-\mathrm{OH}]^{+}$, and minor fragment ions as $[\mathrm{M}+\mathrm{H}-\mathrm{NO}]^{+}$.

Keto-PAHs with fully aromatic ring systems were observed in negative mode as radical anions $[\mathrm{M} \cdot]^{-}$. Cyclopenta[cd]pyrene-3[4H]-one was detected as deprotonated molecule $[\mathrm{M}-\mathrm{H}]^{-}$probably due to the presence of a partially hydrogenated ring system [42]. In the negative ion mode, the tandem MS was dominated by elimination of $\mathrm{H}_{2} \mathrm{CO}$ and a minor fragment ion as a result of losing $\mathrm{H}_{2} \mathrm{O}$ and $\mathrm{CH}_{3}$ [42]. In the positive ion mode, all the keto compounds were detected as protonated molecules $[\mathrm{M}+\mathrm{H}]^{+}$, and the tandem MS fragmentation was attributed to loss of CO [40].

Nitroketo-PAHs were detected in negative ion mode as radical anions $[\mathrm{M} \cdot]^{-}$, while tandem MS resulted in $\left[\mathrm{M}-\mathrm{NO}^{-}\right.$as main fragment ion and minor fragments as $\left[\mathrm{M}-\mathrm{CO}^{-}\right.$. Nitroketo-PAHs were detected in positive ion mode as $\left[\mathrm{M}+\mathrm{H}^{+}\right.$, and the tandem MS resulted in $[\mathrm{M}+\mathrm{H}-\mathrm{OH}]^{+},\left[\mathrm{M}+\mathrm{H}-\mathrm{NO}_{2}\right]^{+}$, and $[\mathrm{M}+\mathrm{H}-\mathrm{NO}]^{+}$.

In negative ion mode, hydroxylated-PAHs were observed as $[\mathrm{M}-\mathrm{H}]^{-}$and tandem MS showed the elimination of $\mathrm{CO}$ as main fragment [40, 42], and the loss of $\mathrm{H}_{2} \mathrm{O}$ as minor fragment. In the positive ion mode, hydroxylated-PAHs were observed as $[\mathrm{M}+\mathrm{H}]^{+}$, and significant signals as result of radical cation $[\mathrm{M} \cdot]^{+}$ for large hydroxides were detected as reported by Letzel et al. $[42,43,44]$. The tandem MS of $[\mathrm{M}+\mathrm{H}]^{+}$ showed the loss of $\mathrm{H}_{2} \mathrm{O}, \mathrm{CO}$ and $\mathrm{H}$ [42], and a minor fragment $[\mathrm{M}+\mathrm{H}-\mathrm{OH}]^{+}$.

In negative ion mode, the molecular anion $[\mathrm{M} \cdot]^{-}$of quinones was the dominating signal [42]. 2-
Methylanthraquinone showed additional $[\mathrm{M}-\mathrm{H}]^{-}$ ions with about $12 \%$ intensity due to the presence of methyl substituent [40]. Tandem MS showed the loss of $[\mathrm{M}-\mathrm{CO}]^{-},\left[\mathrm{M}-\mathrm{H}_{2} \mathrm{O}\right]^{-},\left[\mathrm{M}-\mathrm{CO}_{2}\right]^{-}$, and $\left[\mathrm{M}-\mathrm{CH}_{3}\right]^{-}$ $[40,42]$. In positive ion mode, all the quinones were detected as protonated molecule $[\mathrm{M}+\mathrm{H}]^{+}[43]$, and the major fragment ions were $\left[\mathrm{M}+\mathrm{H}-\mathrm{CO}^{+},[\mathrm{M}+\mathrm{H}-\right.$ $2 \mathrm{CO}^{+}[42]$, and minor fragment ion as $[\mathrm{M}+\mathrm{H}-$ $\mathrm{H}_{2} \mathrm{O}^{+}$. An exceptional case in positive ion mode tandem MS 2-methylanthraquinone showed additional fragment ions: $\left[\mathrm{M}+\mathrm{H}-\mathrm{H}_{2}\right]^{+},\left[\mathrm{M}+\mathrm{H}-\mathrm{HCO}^{+},[\mathrm{M}+\right.$ $\mathrm{H}-2 \mathrm{HCO}^{+},\left[\mathrm{M}+\mathrm{H}-, \mathrm{CO}_{2}\right]^{+},\left[\mathrm{M}+\mathrm{H}-\mathrm{HCO}_{2}\right]^{+}$. Also benz $[a]$ anthracene-7,12-dione gave two additional fragment ions $\left[\mathrm{M}+\mathrm{H}-\mathrm{CO}_{2}\right]^{+}$and $[\mathrm{M}+\mathrm{H}-\mathrm{OH}]^{+}$. The behavior of benz $[a]$ anthracene-7,12-dione may be due to the presence of bay region in the molecular structure.

For hydroxyquinones in negative ion mode, 2hydroxyanthraquinone and 1,3-dihydroxyanthraquinone behaved similarly to hydroxy-PAHs by forming deprotonated molecules $[\mathrm{M}-\mathrm{H}]^{-}$while no molecule radical anion was observed. For 1-hydroxyanthraquinone and 1,8-dihydroxyanthraquinone, molecules with intramolecular H-bonds, the main MS signal arose from the molecule radical anion $[\mathrm{M} \cdot]^{-}[44,45]$. Only minor signals for deprotonated molecules $[\mathrm{M}-\mathrm{H}]^{-}$were observed. In tandem MS, the loss of CO was predominant. In the positive ion mode, hydroxyanthraquinones were observed as $[\mathrm{M}+\mathrm{H}]^{+}$, and the tandem MS fragmentation showed major fragment ions as results of $\left[\mathrm{M}+\mathrm{H}-\mathrm{CO}^{+},\left[\mathrm{M}+\mathrm{H}-2 \mathrm{CO}^{+},\left[\mathrm{M}+\mathrm{H}-\mathrm{C}_{2} \mathrm{O}_{3}\right]^{+}\right.\right.$, and minor fragment ions as result of $\left[\mathrm{M}+\mathrm{H}-\mathrm{H}_{2} \mathrm{O}\right]^{+}$, $\left[\mathrm{M}+\mathrm{H}-\mathrm{CO}_{2}\right]^{+}$, and $\left[\mathrm{M}+\mathrm{H}-3 \mathrm{CO}^{+}\right.$.

Azaarene precursors were observed in positive mode as protonated molecule $[\mathrm{M}+\mathrm{H}]^{+}[46]$ except carbazole and $\mathrm{N}$-Phenyl-2-naphthylamine which were detected also in the negative mode as $[\mathrm{M}-\mathrm{H}]^{-}$. In tandem MS, azaarenes showed the loss of $\mathrm{HCN}$, and/or $\mathrm{H}_{2} \mathrm{CN}$ and $\mathrm{NH}$.

Amino-PAHs precursors were detected in the positive ion mode as $[\mathrm{M}+\mathrm{H}]^{+}[41,47]$. The tandem mass spectra were characterized by signals originating from $\left[\mathrm{M}+\mathrm{H}-\mathrm{NH}_{2}\right]^{+},\left[\mathrm{M}+\mathrm{H}-\mathrm{NH}_{3}\right]^{+},\left[\mathrm{M}+\mathrm{H}-\mathrm{HCN}^{+}\right.$, and $[\mathrm{M}+\mathrm{H}-\mathrm{H}]^{+}[41,47]$. In the negative ion mode, amino-PAHs were detected as $[\mathrm{M}-\mathrm{H}]^{-}$, and in tandem MS mono amino-PAHs were observed as [M $\mathrm{H}-\mathrm{HCN}^{-}$, or $\left[\mathrm{M}-\mathrm{H}-\mathrm{H}_{2} \mathrm{CN}\right]^{-}$, and minor fragment as $[\mathrm{M}-\mathrm{H}-\mathrm{NH}]^{+}$. Diamino-PAHs showed distinctive fragment ion as $\left[\mathrm{M}+\mathrm{H}-\mathrm{H}_{2}-\mathrm{H}_{2} \mathrm{CN}\right]^{+}$.

CID in the LIT part of the hybrid MS resulted only in low intensity fragment spectra for quinones, hydroxyPAHs, and keto-PAHs in the negative mode. Transfer of

Figure 4. Total ion and selected extracted ion chromatograms for the tentative identified major masses in the three selected mutagenic fractions. (a) F1 (upper trace: TIC (-ion mode), and lower trace: EIC $\mathrm{m} / \mathrm{z}$ 230.0740; A1); (b) F2 (upper trace: TIC (-ion mode), and lower trace: EIC $\mathrm{m} / \mathrm{z} 297.0792$; B1); (c) F3 (upper trace: TIC (+ion mode), middle trace: TIC (-ion mode), and lower trace: EIC $\mathrm{m} / \mathrm{z}$ 272.0841; C1). Blank peaks are labeled with an asterisk. 
the latter ions in the ICR cell was not sufficient for recording HR-MS fragment spectra. Therefore, CID fragment spectra were recorded only in the lowresolution LIT.

\section{Method Evaluation on Sediment Extract Fractions}

The tentative identification approach developed in this work for the toxic fractions can be systematically described as shown in Figure 3. The applicability of the method was validated with three mutagenic sediment fractions as complex mixture of unknown compounds with partial polar PACs as major constituents (Figure 4). The selected sediment sample was extracted, and cleaned by using a semipermeable membrane as reported in more details in reference [37, 38]. The dialysate was fractionated on three preparative normal phase columns, and the polar fractions that exhibited strong mutagenic effects in the biotest were further fractionated by reversed-phase RP-HPLC. Tertiary fractionation steps of the active RP-LC fractions were also done using a pyrenyl-bonded silica stationary phase, i.e., the most part of the matrix should be eliminated.

As an example to highlight the tentative identification steps, we select one constituent A1, which was detected in the three fractions (Table 3), eluting after $34.9 \mathrm{~min}$ and detected as $[\mathrm{M} \cdot]^{-}$in the negative mode and as $[\mathrm{M}+\mathrm{H}]^{+}$in the positive mode. The extracted ion chromatogram of the radical anion $[\mathrm{M} \cdot]^{-}$is displayed in Figure 4a (lower trace). The generated empirical formula $\mathrm{C}_{17} \mathrm{H}_{10} \mathrm{O}$ is also deduced from the detected $[\mathrm{M} \cdot]^{-}$, and the MS/MS diagnostic fragment ions [M $\mathrm{CO}^{-},\left[\mathrm{M}-\mathrm{H}_{2} \mathrm{O}\right]^{-},\left[\mathrm{M}-\mathrm{HCO}^{-}\right.$, and $\left[\mathrm{M}-\mathrm{CH}_{3}\right]^{-}$ (Table 3). The database search retrieved 35 hits for this empirical formula (Table S-2 (A1), Supporting Information; possible candidates are shown). Based on the behavior of model compounds, we suggest that the structure is related to keto-PAH with fully aromatic structure. Refining the hits to keto-PAHs with fully aromatic structure reduces the number to 13 [Table S-2 A1; bold font)]. Comparing the retention time and MS/ MS fragmentation pattern with some available candidates showed a perfect match with benzo[a]phenalen7-one. Therefore, compound A1 was unequivocally assigned as benzo[a]phenalen-7-one. TIC for fraction F1 is displayed in Figure 4a; upper trace, the compounds 1,6dinitropyrene assigned by number 1, 3-nitrobenzanthrone assigned by number 2, 6-nitrobenzo[a]pyrene assigned by number 3, 1-nitropyrene assigned by number 4 , and 1,6-pyrenequinone assigned by number 5 were positively identified by reference standards.

The TIC of sediment fraction F2 is displayed in Figure $4 \mathrm{~b}$ upper trace. The unknown B1 behaved in the ion source in the same way as model nitro-PAHs that were used for method development. B1 was observed as radical anion in addition to the loss of $\mathrm{NO}$ in full scan negative ion mode. Extracted mass $(\mathrm{m} / \mathrm{z} 297.0792)$ is shown in Figure $4 \mathrm{~b}$ lower trace. The tandem MS showed a diagnostic neutral loss of NO (Table 3). The database search for generated empirical formula retrieved 38 hits. Considering only nitro compounds the number of hits was reduced to 21 [Table S-2 (B1)]. B1 eluted at $43.5 \mathrm{~min}$ and thus after 6-nitrobenzo[ $a$ ]pyrene (42.6 min). After considering Log $\mathrm{P}$ for the possible structures and the existence of a bay region as shown in Table S-2 we could reduce the number of hits to two. Furthermore, 1,6-pyrenequinone (see 1 in Figure 4b; upper trace) was positively identified by comparison with an authentic standard compound.

Another example is C1 in F3 (see TIC in Figure 4c; upper and middle traces), which showed the same ionization and fragmentation behavior as the methylated quinone used as model compounds. It was observed in the negative mode as radical anion and additionally less intense signals for the deprotonated molecules were detected. The extracted ion chromatogram of the radical anion in negative ion mode is depicted in Figure $4 \mathrm{c}$ lower trace. The tandem MS showed the loss of $\mathrm{H}_{2}, \mathrm{CH}_{3}, \mathrm{H}_{2} \mathrm{O}, \mathrm{CO}, \mathrm{CO}_{2}, \mathrm{C}_{2} \mathrm{H}_{2} \mathrm{O}, 2 \mathrm{CO}$ (Table 3). Considering methylated quinones, the database search retrieved eight possible hits among 97 [Table S-2 (C1)]. The compounds 1,8-dinitropyrene (number 1 in Figure 4c; middle trace), cyclopenta[cd]pyrene$3[4 \mathrm{H}]$-one (number 2), 7-nitrobenz[a]anthracene (number 3), and 1,6-pyrenequinone (number 4) were positively identified by comparison with standard compounds. In summary, the approach discussed for the three examples of sediment extract fractions allowed a tentative identification of several unknown compounds in the three selected fractions with similar functionalities to the selected model compounds (Table 3, Table S-2).

The cleanup protocol and the fractionations prior the identification step improve the robustness of the method from matrix influence (e.g., retention time shift, ionization efficiency). Confirmation and definite assignment of structures by LC-MS is dependent on the availability of commercial standards. In case of absence of reference compounds, synthesis of the respective substances and/or the use of complementary techniques such as GC-MS may be useful.

\section{Conclusions}

A novel methodology integrating database search with diagnostic classifiers from APCI-MS and chromatographic behavior has been developed to simplify the identification of unknown PACs in complex mixtures even without specific reference substances. The applicability of the method was demonstrated by positive and tentative identification in mutagenic sediment fractions with constituents similar to selected model compounds. PubChem database demonstrated a significant potential for tentative identification of toxicants in environmental analysis. However, the discussed approach largely depends on the content of the database used. Hence, compounds which are not present in the database will remain challenging for identification. A major requirement for the future will be to extend 
Table 3. Chromatographic and mass spectrometric data of tentatively identified compounds in the three selected mutagenic sediment fractions

\begin{tabular}{|c|c|c|c|c|c|c|c|c|c|}
\hline & Ion & Observed $\mathrm{m} / \mathrm{z}$ & Rt [min] & EF & MS/MS (+ mode) & aHits & MS classifiers & ${ }^{\mathrm{b} H i t s}$ & Tentative identification \\
\hline \multicolumn{10}{|l|}{$\mathrm{F}-1$} \\
\hline A1 & {$\left[\mathbf{M}^{\cdot}\right]^{-},[\mathrm{M}+\mathrm{H}]^{+}$} & 230.0740 & 34.9 & $\mathrm{C}_{17} \mathrm{H}_{10} \mathrm{O}$ & 203, 216, 199, 189, 216, 202 & 35 & Full aromatic keto-PAHs & 13 & benzo[a]phenalen-7-one \\
\hline A2 & {$\left[\mathbf{M}^{\prime}\right]^{-},[\mathrm{M}+\mathrm{H}]^{+}$} & 232.0530 & 21.1 & $\mathrm{C}_{16} \mathrm{H}_{8} \mathrm{O}_{2}$ & $217,205,190,177,213,233$ & 11 & Quinone & 10 & 1,8-pyrenequinone \\
\hline A3 & {$\left[\mathbf{M}^{-}\right]^{-},[\mathrm{M}+\mathrm{H}]^{+}$} & 244.0892 & 33.8 & $\mathrm{C}_{18} \mathrm{H}_{12} \mathrm{O}$ & $203,227,217,230,245$ & 108 & Full aromatic keto-PAHs & 16 & 1-acetylpyrene \\
\hline \multicolumn{10}{|c|}{$\mathrm{F}-2$} \\
\hline B1 & {$\left[\mathbf{M}^{\cdot}\right]^{-},[\mathrm{M}-\mathrm{NO}]^{-}$} & 297.0792 & 43.5 & $\mathrm{C}_{20} \mathrm{H}_{11} \mathrm{NO}_{2}$ & ${ }^{\mathrm{c}} \mathbf{2 6 7}, 282$ & 38 & Full aromatic Nitro-PAHs & 21 & 1-nitrobenzo[e]pyrene \\
\hline B2 & {$\left[\mathbf{M}^{\cdot}\right]^{-},[\mathrm{M}+\mathrm{H}]^{+}$} & 278.0733 & $41.8,43.5,43.9$ & $\mathrm{C}_{21} \mathrm{H}_{10} \mathrm{O}$ & $251,261,237$ & 4 & Full aromatic keto-PAHs & 3 & $11 \mathrm{H}$-cyclopenta(ghi)perylen-11-one \\
\hline B3 & {$\left[\mathbf{M}^{-}\right]^{-},[\mathrm{M}+\mathrm{H}]^{+}$} & 247.0639 & 41.2 & $\mathrm{C}_{16} \mathrm{H}_{9} \mathrm{NO}_{2}$ & c217, 227 & 93 & Full aromatic Nitro-PAHs & 10 & 1-nitrofluoranthene \\
\hline B4 & {$\left[\mathbf{M}^{-}\right]^{-},[\mathbf{M}+\mathbf{H}]^{+}$} & 277.0892 & $40.7,41.9,42.5$ & $\mathrm{C}_{21} \mathrm{H}_{11} \mathrm{~N}$ & $277,251,278,263,262,242,222$ & 4 & Cyano-PAHs & 3 & benzo[a]pyrene-6-carbonitrile \\
\hline A1 & {$\left[\mathbf{M}^{-}\right]^{-},[\mathrm{M}+\mathrm{H}]^{+}$} & 230.0740 & 35.0 & $\mathrm{C}_{17} \mathrm{H}_{10} \mathrm{O}$ & $203,216,199,189,216,202$ & 35 & Full aromatic keto-PAHs & 13 & benzo[a]phenalen-7-one \\
\hline A2 & {$\left[\mathbf{M}^{\cdot}\right]^{-},[\mathrm{M}+\mathrm{H}]^{+}$} & 232.0530 & 21.1 & $\mathrm{C}_{16} \mathrm{H}_{8} \mathrm{O}_{2}$ & $217,205,190,177,213,233$ & 11 & Quinone & 10 & 1,8-pyrenequinone \\
\hline \multicolumn{10}{|c|}{$\mathrm{F}-3$} \\
\hline $\mathrm{C} 1$ & $\begin{array}{l}{\left[\mathbf{M}^{*}\right]^{-},[\mathrm{M}-\mathrm{H}]^{-}} \\
{[\mathrm{M}+\mathrm{H}]^{+}}\end{array}$ & 272.0841 & $33.6,34.3$ & $\mathrm{C}_{19} \mathrm{H}_{12} \mathrm{O}_{2}$ & $231,245,229,255,217$ & 97 & Methylated quinone & 8 & $\begin{array}{l}\text { 7-methylbenzo[a]anthracene-3,4- } \\
\text { dione }\end{array}$ \\
\hline $\mathrm{C} 2$ & {$[\mathbf{M}+\mathbf{H}]^{+}$} & 230.0966 & 34.5 & $\mathrm{C}_{17} \mathrm{H}_{12} \mathrm{~N}$ & $230,202,213,212,215$ & 61 & Azaarenes & 29 & 1-azabenz[a]anthracene \\
\hline $\mathrm{C} 3$ & {$[\mathbf{M}+\mathbf{H}]^{+}$} & 254.0966 & 36.4 & $\mathrm{C}_{19} \mathrm{H}_{12} \mathrm{~N}$ & $254,253,226,227,238,219,212$ & 29 & Azaarenes & 20 & 2-azabenzo[b]pyrene \\
\hline $\mathrm{C} 4$ & {$[\mathrm{M}+\mathrm{H}]^{+},[\mathrm{M}]^{-}$} & 255.0806 & 35.9 & $\mathrm{C}_{19} \mathrm{H}_{11} \mathrm{O}$ & $227,226,240,237,199,255$ & 6 & Full aromatic keto-PAHs & 6 & 4,5-oxochrysene \\
\hline C5 & {$\left[\mathbf{M}^{\cdot}\right]^{-},[\mathrm{M}+\mathrm{H}]^{+}$} & 282.0686 & $33.9,36.1$ & $\mathrm{C}_{20} \mathrm{H}_{10} \mathrm{O}_{2}$ & $227,255,265,239,383,211$ & 29 & Quinone with bay region & 6 & benzo[a]pyrene-11,12-dione \\
\hline A1 & {$\left[\mathbf{M}^{\cdot}\right]^{-},[\mathrm{M}+\mathrm{H}]^{+}$} & 230.0740 & 34.9 & $\mathrm{C}_{17} \mathrm{H}_{10} \mathrm{O}$ & 203, 216, 199, 189, 216, 202 & 35 & Full aromatic keto-PAHs & 13 & benzo[a]phenalen-7-one \\
\hline A2 & {$\left[\mathbf{M}^{\cdot}\right]^{-},[\mathrm{M}+\mathrm{H}]^{+}$} & 232.0530 & 21.1 & $\mathrm{C}_{16} \mathrm{H}_{8} \mathrm{O}_{2}$ & $217,205,190,177,213,233$ & 11 & Quinone & 10 & 1,8-pyrenequinone \\
\hline A3 & {$\left[\mathbf{M}^{\cdot}\right]^{-},[\mathrm{M}+\mathrm{H}]^{+}$} & 244.0892 & $33.9,34.7$ & $\mathrm{C}_{18} \mathrm{H}_{12} \mathrm{O}$ & $203,217,227,230,245$ & 108 & Full aromatic keto-PAHs & 16 & 4-acetylpyrene \\
\hline
\end{tabular}

Bold font indicates high relative intensity.

aNo. of database hits using the empirical formula (EF) as searching criterion.

bossible hits after reduction based on MS spectral classifiers.

'Tandem MS for the precursor ion in the negative mode. 
available classifiers and the compound groups with different structural isomers for which they are valid. In particular, with respect to chromatographic behavior of isomers on different stationary phases, in addition to $\log \mathrm{P}$, only very limited classifiers are available. Models relating the structure of a compound and its retention are still limited to the classical Log P-based approach, and the application of linear free-energy relationships according to Abraham parameters [8], which is considering more specific interactions such as $\mathrm{H}$-bonding shows promise for future applications. It is evident that steric properties play an important role for the retention of analytes on stationary phases. To include these properties in retention modeling would be an important step towards powerful chromatographic classifiers.

\section{Acknowledgments}

The authors acknowledge support for this research by the European Commission through the Marie Curie Research Training Network KEYBIOEFFECTS (contract-no. MRTN-CT-2006-035695), and the Integrated Project MODELKEY (contract no. 511237GOCE). The Ministerium für Innovation, Wissenschaft, Forschung und Technologie des Landes Nordrhein-Westfalen, (Düsseldorf, Germany) and the Bundesministerium für Bildung und Forschung, (Bonn, Germany) are gratefully acknowledged for funding the parts of the research carried out at the ISAS. The authors thank Ms. Helma Geltenpoth for the excellent technical support.

\section{Appendix A Supplementary Material}

Supplementary material associated with this article may be found in the online version at doi:10.1016/ j.jasms.2010.02.015.

\section{References}

1. Belpomme, D.; Irigaray, P.; Hardell, L.; Clapp, R.; Montagnier, L.; Epstein, S.; Sasco, A. J. The Multitude and Diversity of Environmental Carcinogens. Environ. Res. 2007, 105(3), 414-429.

2. Finlayson-Pitts, B. J.; Pitts, J. J. N. Airborne Polycyclic Aromatic Hydrocarbons and Their Derivatives: Atmospheric Chemistry and Toxicological Implications. In Chemistry of the Upper and Lower Atmosphere; Academic Press: San Diego, 2000; p 436.

3. Kim, P. M.; DeBoni, U.; Wells, P. G. Peroxidase-Dependent Bioactivation and Oxidation of DNA and Protein in Benzo[a]Pyrene-Initiated Micronucleus Formation. Free Rad. Biol. Med. 1997, 23(4), 579-596.

4. Brack, W. Effect-Directed Analysis: A Promising Tool for the Identification of Organic Toxicants in Complex Mixtures? Anal. Bioanal. Chem. 2003, 377(3), 397-407.

5. Brack, W.; Klamer, H. J. C.; de Ada, M. L.; Barcelo, D. Effect-Directed Analysis of Key Toxicants in European River Basins-a review. Environ. Sci. Poll. Res. 2007, 14(1), 30-38.

6. Hewitt, L. M.; Marvin, C. H. Analytical Methods in Environmental Effects-Directed Investigations of Effluents. Mut. Res. 2005, 589(3), $208-232$.

7. Brack, W.; Schmitt-Jansen, M.; Machala, M.; Brix, R.; Barcelo, D.; Schymanski, E.; Streck, G.; Schulze, T. How to Confirm Identified Toxicants in Effect-Directed Analysis. Anal. Bioanal. Chem. 2008, 390(8), 1959-1973.

8. Schymanski, E. L.; Bataineh, M.; Goss, K. U.; Brack, W. Integrated Analytical and Computer Tools for Structure Elucidation in EffectDirected Analysis. TrAC Trends Anal. Chem. 2009, 28(5), 550-561.

9. Matsushita, T.; Matsui, Y.; Matsui, Y. Estimating Mutagenic Compounds Generated During Photolysis of Fenitrothion-by HPLC Fractionation Followed by Mutagenicity Testing and High-Resolution GC-MS Analysis. Chemosphere 2006, 64(1), 144-151.

10. Watanabe, T.; Ohba, H.; Asanoma, M.; Hasei, T.; Takamura, T.; Terao, Y.; Shiozawa, T.; Hirayama, T.; Wakabayashi, K.; Nukaya, H. Isolation and Identification of Nonchlorinated Phenylbenzotriazole (nonCIPBTA)-Type Mutagens in the Ho River in Shizuoka Prefecture, Japan. Mutat. Res.-Genet. Toxicol. Environ. Mutagenesis 2006, 609(2), 137-145.

11. Kami, H.; Watanabe, T.; Takemura, S.; Kameda, Y.; Hirayama, T Isolation and Chemical-Structural Identification of a Novel Aromatic Amine Mutagen in an Ozonized Solution of m-Phenylenediamine. Chem. Res. Toxicol. 2000, 13(3), 165-169.

12. Grung, M.; Lichtenthaler, R.; Ahel, M.; Tollefsen, K. E.; Langford, K.; Thomas, K. V. Effects-Directed Analysis of Organic Toxicants in Wastewater Effluent from Zagreb, Croatia. Chemosphere 2007, 67(1), 108-120.

13. Brack, W.; Schirmer, K.; Erdinger, L.; Hollert, H. Effect-Directed Analysis of Mutagens and Ethoxyresorufin-O-Deethylase Inducers in Aquatic Sediments. Environ. Toxicol. Chem. 2005, 24(10), 2445-2458.

14. Houtman, C. J.; Van Oostveen, A. M.; Brouwer, A.; Lamoree, M. H.; Legler, J. Identification of Estrogenic Compounds in Fish Bile Using Bioassay-Directed Fractionation. Environ. Sci. Technol. 2004, 38(23), 6415-6423.

15. Bailey, H. C.; Elphick, J. R.; Krassoi, R.; Mulhall, A. M.; Lovell, A. J.; Slee, D. J. Identification of Chlorfenvinphos Toxicity in a Municipal Effluent in Sydney, New South Wales, Australia. Environ. Toxicol. Chem. 2005, 24(7), 1773-1778.

16. Castillo, M.; Barcelo, D. Characterization of Organic Pollutants in Textile Wastewaters and Landfill Leachate by Using Toxicity-Based Fractionation Methods Followed by Liquid and Gas Chromatography Coupled to Mass Spectrometric Detection. Anal. Chim. Acta 2001, 426(2), 253-264.

17. Biselli, S.; Reineke, N.; Heinzel, N.; Kammann, U.; Franke, S.; Huhnerfuss, H.; Theobald, N. Bioassay-Directed Fractionation of Organic Extracts of Marine Surface Sediments from the North and Baltic Sea. Part I: Determination and Identification of Organic Pollutants. J. Soils Sediments 2005, 5(3), 171-181.

18. Thomas, K. V.; Hurst, M. R.; Matthiessen, P.; Waldock, M. J. Characterization of Estrogenic Compounds in Water Samples Collected from United Kingdom Estuaries. Environ. Toxicol. Chem. 2001, 20(10), 21652170 .

19. Thomas, K. V.; Balaam, J.; Barnard, N.; Dyer, R.; Jones, C.; Lavender, J.; McHugh, M. Characterization of Potentially Genotoxic Compounds in Sediments Collected from United Kingdom Estuaries. Chemosphere 2002, 49(3), 247-258.

20. Koh, C. H.; Khim, J. S.; Villeneuve, D. L.; Kannan, K.; Giesy, J. P Analysis of Trace Organic Contaminants in Sediment, Pore Water, and Water Samples from Onsan Bay, Korea: Instrumental Analysis and In Vitro Gene Expression Assay. Environ. Toxicol. Chem. 2002, 21(9), 1796-1803.

21. Reineke, N.; Bester, K.; Huhnerfuss, H.; Jastorff, B.; Weigel, S. BioassayDirected Chemical Analysis of River Elbe Surface Water Including Large Volume Extractions and High Performance Fractionation. Chemosphere 2002, 47(7), 717-723.

22. Noguchi, K.; Toriba, A.; Chung, S. W.; Kizu, R.; Hayakawa, K. Identification of Estrogenic/Anti-Estrogenic Compounds in Diesel Exhaust Particulate Extract. Biomed. Chromatogr. 2007, 21(11), 1135-1142.

23. Grimalt, S.; Pozo, O. J.; Sancho, J. V.; Hernandez, F. Use of Liquid Chromatography Coupled to Quadrupole Time-of-Flight Mass Spectrometry to Investigate Pesticide Residues in Fruits. Anal. Chem. 2007, 79(7), 2833-2843.

24. Perez, S.; Barcelo, D. First Evidence for Occurrence of Hydroxylated Human Metabolites of Diclofenac and Aceclofenac in Wastewater Using QqLIT-MS and QqTOF-MS. Anal. Chem. 2008, 80(21), 8135-8145.

25. Reemtsma, T.; These, A.; Venkatachari, P.; Xia, X. Y.; Hopke, P. K.; Springer, A.; Linscheid, M. Identification of Fulvic Acids and Sulfated and Nitrated Analogues in Atmospheric Aerosol by Electrospray Ionization Fourier Transform Ion Cyclotron Resonance Mass Spectrometry. Anal. Chem. 2006, 78(24), 8299-8304.

26. Pace, C. M.; Betowski, L. D. Measurement of High-Molecular-Weight Polycyclic Aromatic Hydrocarbons in Soils by Particle Beam HighPerformance Liquid Chromatography-Mass Spectrometry. J. Am. Soc. Mass Spectrom. 1995, 6, 597-607.

27. Bonfanti, L.; Careri, M.; Mangia, A.; Manini, P.; Maspero, M. Simultaneous Identification of Different Classes of Hydrocarbons and Determination of Nitro-Polycyclic Aromatic Hydrocarbons by Means of Particle Beam Liquid Chromatography-Mass Spectrometry. J. Chromatogr. A 1996, 728, 359-369.

28. Moriwaki, H.; Ishitake, M.; Youhikawa, S.; Miyakoda, H.; Alary, J. F. Determination of Polycyclic Aromatic Hydrocarbons in Sediment by Liquid Chromatography-Atmospheric Pressure Photoionization-Mass Spectrometry. Analyt. Sci. 2004, 20, 375-377.

29. Itoh, N.; Aoyagi, Y.; Yarita, T. Optimization of the Dopant for the Trace Determination of Polycyclic Aromatic Hydrocarbons by Liquid Chromatography/Dopant-Assisted Atmospheric-Pressure Photoionization/ Mass Spectrometry. J. Chromatogr. A 2006, 1131, 285-288.

30. Hill, D. W.; Kertesz, T. M.; Fontaine, D.; Friedman, R.; Grant, D. F. Mass Spectral Metabonomics Beyond Elemental Formula: Chemical Database Querying by Matching Experimental with Computational Fragmentation Spectra. Anal. Chem. 2008, 80(14), 5574-5582.

31. Polettini, A.; Gottardo, R.; Pascali, J. P.; Tagliaro, F. Implementation and Performance Evaluation of a Database of Chemical Formulas for the Screening of Pharmaco/Toxicologically Relevant Compounds in Biological Samples Using Electrospray Ionization-Time-of-Flight Mass Spectrometry. Anal. Chem. 2008, 80(8), 3050-3057. 
32. Thurman, E. M.; Ferrer, I.; Fernandez-Alba, A. R. Matching Unknown Empirical Formulas to Chemical Structure Using LC/MS TOF Accurate Mass and Database Searching: Example of Unknown Pesticides on Tomato Skins. J. Chromatogr. A 2005, 1067(1-2), 127-134.

33. Hao, H. P.; Cui, N.; Wang, G. J.; Xiang, B. R.; Liang, Y.; Xu, X. Y.; Zhang, H.; Yang, J.; Zheng, C. N.; Wu, L.; Gong, P.; Wang, W. Global Detection and Identification of Nontarget Components from Herbal Preparations by Liquid Chromatography Hybrid Ion Trap Time-of-Flight Mass Spectrometry and a Strategy. Anal. Chem. 2008, 80(21), 8187-8194.

34. Katajamaa, M.; Oresic, M. Processing Methods for Differential Analysis of LC/MS Profile Data. BMC Bioinformatics 2005, 6:179, 1-12.

35. Katajamaa, M.; Miettinen, J.; Oresic, M. MZmine: Toolbox for Processing and Visualization of Mass Spectrometry-Based Molecular Profile Data. Bioinformatics 2006, 22(5), 634-636.

36. Katajamaa, M.; Oresic, M. Data Processing for Mass SpectrometryBased Metabolomics. J. Chromatoor. A 2007, 1158(1-2), 318-328.

37. Lübcke-von Varel, U.; Streck, G.; Brack, W. Automated Fractionation Procedure for Polycyclic Aromatic Compounds in Sediment Extracts on Three Coupled Normal-Phase High-Performance Liquid Chromatography Columns. J. Chromatogr. A 2008, 1185(1), 31-42

38. Lübcke-von Varel, U. 2009, unpublished (manuscript in preparation).

39. Wise, S. A.; Bonnett, W. J.; Guenther, F. R.; May, W. E. A Relationship Between Reversed-Phase C18 Liquid-Chromatographic Retention and the Shape of Polycyclic Aromatic-Hydrocarbons. I. Chromatogr. Sci. 1981, 19(9), 457-465.

40. Letzel, T.; Poschl, U.; Wissiack, R.; Rosenberg, E.; Grasserbauer, M.; Niessner, R. Phenyl-Modified Reversed-Phase Liquid Chromatography Coupled to Atmospheric Pressure Chemical Ionization Mass Spectrometry: A Universal Method for the Analysis of Partially Oxidized Aromatic Hydrocarbons. Anal. Chem. 2001, 73(7), 1634-1645.
41. Straube, E. A.; Dekant, W.; Volkel, W. Comparison of Electrospray Ionization, Atmospheric Pressure Chemical Ionization, and Atmospheric Pressure Photoionization for the Analysis of Dinitropyrene and Aminonitropyrene LC-MS/MS. J. Am. Soc. Mass Spectrom. 2004, 15(12), 1853-1862.

42. Letzel, T.; Poschl, U.; Rosenberg, E.; Grasserbauer, M.; Niessner, R. In-Source Fragmentation of Partially Oxidized Mono- and Polycyclic Aromatic Hydrocarbons in Atmospheric Pressure Chemical Ionization Mass Spectrometry Coupled to Liquid Chromatography. Rapid Commun. Mass Spectrom. 1999, 13(24), 2456-2468.

43. Letzel, T.; Rosenberg, E.; Wissiack, R.; Grasserbauer, M.; Niessner, R. Separation and Identification of Polar Degradation Products of Benzo [a]Pyrene with Ozone by Atmospheric Pressure Chemical IonizationMass Spectrometry After Optimized Column Chromatographic CleanUp. J. Chromatogr. A 1999, 855(2), 501-514.

44. Galceran, M. T.; Moyano, E. Determination of Hydroxy Polycyclic Aromatic Hydrocarbons by Liquid Chromatography Mass SpectrometryComparison of Atmospheric Pressure Chemical Ionization and Electrospray. J. Chromatogr. A 1996, 731(1-2), 75-84.

45. Grosse, S.; Letzel, T. Liquid Chromatography/Atmospheric Pressure Ionization Mass Spectrometry with Post-Column Liquid Mixing for the Efficient Determination of Partially Oxidized Polycyclic Aromatic Hydrocarbons. J. Chromatogr. A 2007, 1139(1), 75-83.

46. Castillo, M.; Alpendurada, M. F.; Barcelo, D. Characterization of Organic Pollutants in Industrial Effluents Using Liquid Chromatography Atmospheric Pressure Chemical Ionization Mass Spectrometry. J. Mass Spectrom. 1997, 32(10), 1100-1110.

47. Straube, E.; Dekant, W.; Volkel, W. Enhanced Sensitivity for the Determination of Amphiphilic Polyaromatic Amines by LC-MS/MS after Acetylation. J. Chromatogr. A 2005, 1067(1-2), 181-190. 